\title{
Contribuciones para una mirada marxista acerca de los usos de la tecnología bajo el capitalismo
}

\author{
Contributions for a Marxist perspective on the uses of technology under capitalism
}

Contribucions a una mirada marxista sobre els usos de la tecnologia en el capitalisme

\author{
Ilana Lemos de Paiva ${ }^{\circledR}$, Gabriel Miranda*(ْ) \\ Universidade Federal do Rio Grande do Norte, Natal, Brasil \\ *Autor para correspondencia: g.m.b94_@hotmail.com (Gabriel Miranda)
}

Recibido: 31/07/2022 | Aceptado: 06/10/2021 | Publicado: 15/01/2022

\begin{abstract}
RESUMEN: El trabajo es una categoría central para el análisis marxista, siendo considerado indispensable para la reproducción social. Las discusiones sobre el denominado capitalismo digital y los cambios drásticos en el mundo del trabajo traen nuevos desafíos a los investigadores, especialmente después de las fracturas económicas provocadas por la pandemia del nuevo coronavirus. Según la experiencia histórica, el desarrollo tecnológico bajo el capitalismo se caracteriza por la mercantilización de productos derivados de los avances tecnocientíficos, lo que resulta en la exclusión de vastos grupos del acceso a bienes y servicios. Además, también se caracteriza por un conjunto de usos asociados a la precariedad de las condiciones de vida de los trabajadores. Por lo que corresponde a los impactos provocados en el mundo del trabajo, por un lado, los trabajadores se están reduciendo a la informalidad y, por otro, los mecanismos de gestión y control de los procesos de trabajo están perdiendo sus formas reconocidas. En este sentido, este artículo pretende discutir las implicaciones de los avances tecnocientíficos para la vida concreta de los trabajadores ante la contradicción capital-trabajo en el mundo digitalizado. Así, el objetivo es ensayar algunas reflexiones sobre la importancia del proceso formal de enseñanza-aprendizaje, con énfasis en las instituciones de educación superior, para enfrentar los impactos del capitalismo digital. Se asume que la adopción de una mirada marxista en los debates sobre ciencia, tecnología y sociedad es un hito importante en la disputa por la producción de un conocimiento científico comprometido con el bienestar colectivo y mejoras concretas en la vida de los trabajadores.
\end{abstract}

Cómo citar: Lemos de
Paiva, I. y Miranda, G. (2022). Contribuciones para una mirada marxista acerca de los usos de la tecnología bajo el capitalismo.

Research in Education and

Learning Innovation

Archives, 28,50-60.

10.7203/realia.28.21421

Copyright: El/La Autor/a. Open Access: Este es un artículo de acceso abierto distribuido bajo los términos de la licencia Creative Commons

Attribution-NoDerivatives 4.0 International licence (CC BY-ND 4.0)

PALABRAS CLAVE: Marxismo; Relaciones CTS; Tecnología; Educación; Mundo del trabajo

ABSTRACT: Work is central to Marxist analysis, where it is considered indispensable for social reproduction. In the contemporary world, discussions on so-called digital capitalism and the dramatic changes that have taken place in the working environment have brought new challenges for researchers, especially after the economic divisions created by the new worldwide pandemic. Digitization of the working environment and technological progress have been accompanied by precariousness in workers' living conditions. On the one hand, workers are being driven towards informality while, on the other, the mechanisms for managing and controlling work processes are losing their recognized forms. In this article we discuss the impact of techno-scientific advancement on the lives of workers in view of the contradiction between capital and labour in the digital world. Our aim is to offer several reflections on the importance of the formal teaching-learning process, especially in higher education institutions, in order to meet the challenges presented by digital capitalism. We assume the adoption of a Marxist perspective in debates on science, technology and society to be an 
important milestone in the struggle to produce scientific knowledge that is committed to achieving collective well-being and real improvements in workers' lives.

KEYWORDS: Marxism; STS relations; Technology; Education; World of work

RESUM: La de treball és una categoria central de l'anàlisi marxista. El treball es considera indispensable per a la reproducció social. Les discussions sobre el denominat capitalisme digital i els canvis dràstics en el món del treball plantegen nous desafiaments als investigadors, especialment després de les fractures econòmiques que ha provocat la pandèmia del coronavirus. Segons l'experiència històrica, el desenvolupament tecnològic en el capitalisme es caracteritza per la mercantilització de productes derivats dels avanços tecnocientífics, la qual cosa té el resultat que vastos grups de població no tinguen accés a béns i serveis. A més, també es caracteritza per un conjunt d'usos associats a la precarietat de les condicions de vida dels treballadors. Pel que correspon als impactes en el món del treball, d'una banda, els treballadors s'estan reduint a la informalitat i, d'una altra, els mecanismes de gestió i control dels processos de treball estan perdent les seues formes reconegudes. En aquest sentit, aquest article pretén discutir les implicacions dels avanços tecnocientífics per a la vida concreta dels treballadors davant la contradicció capital-treball en el món digitalitzat. Així, l'objectiu és fer algunes reflexions sobre la importància del procés formal d'ensenyament-aprenentatge, amb especial atenció a les institucions d'educació superior, per a enfrontar-se amb els impactes del capitalisme digital. Amb el pressupòsit que adoptar una mirada marxista en els debats sobre ciència, tecnologia i societat és una fita important en la disputa sobre la producció d'un coneixement científic compromés amb el benestar col-lectiu i les millores concretes en la vida dels treballadors.

PARAULES CLAU: Marxisme; Relacions CTS; Tecnologia; Educació; Món del treball

Notas de aplicación práctica

Qué se sabe sobre el tema

- En las sociedades capitalistas, la tecnología adquiere un carácter contradictorio, ya que se puede constituir como sinónimo de progreso o de barbarie.

- Las redes sociales y los móviles son dos mecanismos de escape de una sociedad que se dirige hacia la precarización neoliberal de las condiciones generales de vida.

\section{Qué aporta este trabajo}

- El artículo plantea una reflexión sobre el mundo del trabajo digital, basado en un análisis marxista de la precariedad laboral.

- Se analiza la relación entre tecnología y sociedad, y sus implicaciones en la producción de conocimiento comprometida con el bienestar colectivo.

Implicaciones para la práctica y/o política

- La adopción de una mirada marxista en los debates sobre ciencia, tecnología y sociedad constituye un marco teórico importante para el currículo académico y la formulación de políticas públicas.

- Es necesario construir un debate académico y público que permita impulsar una ciencia alineada con la satisfacción de las necesidades humanas y no con los intereses capitalistas.

- Debido al rol de las tecnologías digitales en la sociedad contemporánea, es necesario contar con políticas que informen a los ciudadanos sobre las maneras como pueden utilizar estas redes sociales y otras aplicaciones móviles.

\section{INTRODUCCIÓN}

Karl Marx (1818-1883), teórico y revolucionario del siglo XIX, dedicó una parte considerable de su vida a comprender el modo de producción capitalista y las relaciones sociales que engendra. Se preocupó, sobre todo, por analizar los problemas que planteaba el capitalismo de su tiempo, aunque ello no implicara un desconocimiento de los procesos históricos pasados ni una falta de compromiso con el futuro. Así, a pesar de que en Marx no hay una reflexión sistematizada sobre el tema de la tecnología, al obser- 
var el desarrollo altamente tecnologizado del capitalismo, que estaba transformando rápidamente el mundo, el autor consideró indispensable incorporar los estudios de la tecnología y las ciencias experimentales de su época a la comprensión de la economía.

En el mundo contemporáneo, los efectos del llamado "capitalismo digital" han sido considerados desde diferentes perspectivas, al ser abordados en estudios que van desde el debate sobre la automatización/robotización de la producción, la inteligencia artificial, la industria 4.0, hasta las discusiones más actuales sobre los trabajadores de plataformas online, la gig economy y los riesgos de la informalidad del trabajo bajo demanda.

Es cierto que, ante las reconfiguraciones del capitalismo contemporáneo, está en marcha otro cambio drástico en el mundo del trabajo, con diversas consecuencias para la vida concreta de los trabajadores. Hoy en día, Marx tendría que preguntarse: ¿cuáles son los desafíos para el trabajo y para un proyecto de emancipación humana frente a los actuales avances de la tecnología y la comunicación? Y, más concretamente, en un mundo fracturado por la pandemia de Covid-19, ¿cuáles son los debates que se abren ante una serie de tendencias explotadoras del capitalismo digital contemporáneo? ¿Tiene algún sentido desvelar tales procesos sobre la base de Marx y la teoría del trabajo del valor, incluso frente a las ideas que han propagado en las últimas décadas relacionadas con "el fin del trabajo"?

La afirmación de que Marx es un pensador que no puede responder a los desafíos de la época actual no resiste a una rápida mirada al reino de la miseria en que se ha transformado el mundo en que vivimos. La concentración de la riqueza, por un lado, y el aumento abismal de la desigualdad y la pobreza, por otro, son dos caras del mismo proceso brutal de expansión del capitalismo. Como no se trata de crisis esporádicas, los teóricos marxistas llevan tiempo diciendo que estamos inmersos en una crisis sistémica del capitalismo que involucra toda la estructura del orden del Capital (Chesnais, 2016; Mészáros, 2009). Por tanto, la pregunta que hay que hacerse es ¿cómo entender la precarización de la vida de miles de trabajadores en el mundo actual del capital global y digitalizado sin recurrir a Marx?

En este sentido, este artículo se estructura en cuatro secciones. En la primera, se discute acerca de la centralidad del trabajo y la importancia de la relación valor-trabajo como forma de dominación social. A su vez, la segunda sección trata sobre la contradicción capital-trabajo en el mundo digitalizado y los desafíos para los investigadores que buscan comprender lo que se ha llamado uberización del trabajo. Por fin, en la tercera sección es presentado el carácter dialéctico de la tecnología bajo el capitalismo, y, en la cuarta, son planteadas algunas contribuciones acerca del papel asumido por la educación para construcción de trabajadores que puedan posicionarse críticamente ante la tecnología y los imperativos del capital.

\section{I}

Recordemos que Marx señaló de manera radical la historicidad de la vida humana. Su interés se dirigía a la comprensión de los procesos que engendran el cambio social, dilucidando la formación de relaciones sociales de producción que corresponden a una etapa definida de desarrollo de las fuerzas materiales de producción. Evidentemente, en vista de su vasta obra, Marx estuvo expuesto a numerosas interpretaciones. Para Lukács (2004), Marx entiende la idea de la constitución del ser social a partir de la categoría del trabajo:

El trabajo, que en un principio puede verse como algo meramente instintivo del ser humano para su supervivencia, representa, en realidad, un salto ontológico -y cualitativo- que marca el paso de la esfera biológica a la esfera del ser social, constituyéndose como elemental 
para otras dimensiones de la vida, como la historia, la sociabilidad y el lenguaje, ya que el trabajo "marca sobre todo el paso, en el hombre que trabaja, del ser meramente biológico al ser social.

$$
\text { (2004, p. 44) }
$$

Es a través del trabajo que los sujetos se desprenden de la naturaleza. Es decir, la interacción entre los seres humanos y la naturaleza es y produce la socialización del ser social y los cambios cuantitativos y cualitativos en las relaciones sociales y de producción. Así, hay que buscar en el ser social su lógica específica, la procesualidad ontológica por la que se diferencia del ser puramente natural. En este sentido, el trabajo es, por tanto, un punto de cruce entre las interrelaciones de las legalidades de la naturaleza y de la sociedad, cuya característica ontológica es la de poner en los contornos puramente sociales todo lo que se deriva de este proceso

Es a través del trabajo que el ser social produce y reproduce sus condiciones de vida, lo que implica dimensiones objetivas y subjetivas de su existencia. Mucho más que ser la fuente de toda riqueza, el trabajo se constituye como una condición básica y fundamental de toda vida humana (Schmidt, 1977). Marx y Engels afirman:

[...] el primer presupuesto de toda existencia humana, y, por tanto, de toda historia, es que todos los hombres deben estar en condiciones de vivir para hacer historia. Pero para vivir es necesario, en primer lugar, comer, beber, vivir, vestirse y algunas otras cosas. El primer hecho histórico es, por lo tanto, la producción de los medios para satisfacer estas necesidades, la producción de la vida material misma; y esto mismo constituye un hecho histórico, una condición fundamental de toda la historia que debe, aún hoy como hace miles de años, cumplirse día a día, hora a hora, simplemente para mantener a los hombres vivos.

(2014, p. 21, )

Así, partiendo de esta comprensión, no se puede hablar del "fin del trabajo", ya que, incluso con los avances tecnológicos, el trabajo humano es indispensable para la reproducción social y para el desarrollo de tecnologías cada vez más avanzadas (Lukács, 1985). Según Marx:

Este proceso de trabajo concreto busca mejorar los elementos naturales a las necesidades humanas - y es condición natural eterna de la vida humana

$$
\text { (1973, p. 208) }
$$

En el capitalismo, las fuerzas productivas adquieren nuevas dimensiones y, como sabemos, el trabajador es reducido a su fuerza de trabajo (Wittmann, 2009). En este modo de producción hay dos características fundamentales: la producción de mercancías - para la que el valor-trabajo es fundamental - y la producción de ganancia, que se genera con la producción de plusvalor (o plusvalía), como lo afirma el propio Marx:

El proceso de producción, cuando es una unidad del proceso de trabajo y del proceso de producción de valor, es un proceso de producción de mercancías; cuando es una unidad del proceso de trabajo y del proceso de producción de plusvalía, es un proceso capitalista de mercancías, una forma capitalista de producción de mercancías. 
El trabajo humano es lo único capaz de generar valor, y es indispensable para el modo de producción capitalista. La pandemia del nuevo coronavirus ha hecho aún más evidente esta característica, dada la necesidad de mantener en casa a los trabajadores de diversos sectores como medida de prevención sanitaria.

En la producción capitalista, el proceso de trabajo que interesa investigar es el de la producción de mercancías, porque en el capitalismo las mercancías son la forma elemental de riqueza. Al examinarlo, uno se da cuenta de que hay una contradicción privada-social vinculada al trabajo. Es decir, el trabajo es privado, pero está sometido a una división social, de los productos del trabajo y de las tareas para producirlo. La solución que encuentra el modo de producción capitalista está en el intercambio, pasando por el valor y el dinero como relaciones típicas del capitalismo. Por lo tanto, los trabajadores necesitan producir valores de uso para otros, y lo que se vuelve importante es el trabajo abstracto que crea el valor de las mercancías.

El valor tiene un contenido cualitativo, una sustancia, que es el trabajo abstracto, pero también tiene un contenido cuantitativo, que es el trabajo socialmente necesario para producir las mercancías. Cuando las mercancías se convierten en dinero, se verifican varias transformaciones en las relaciones sociales de producción.

La generalización de la producción de mercancías se completa con la transformación de la fuerza de trabajo, es decir la capacidad humana de trabajo, en mercancía. Todo este proceso genera una coerción económica sobre la clase que sólo posee su fuerza de trabajo para sobrevivir, ya que es indispensable comprar los artículos necesarios para la reproducción de la vida, como señaló el propio Marx. ¿Cómo se produce esa coacción en el capitalismo?

La nueva clase que ascendió con la revolución burguesa mantuvo su poder y hegemonía a través del beneficio y sus usos, estableciendo una contradicción esencial para el modelo productivo emergente. Por un lado, la necesidad imperiosa de ampliar el beneficio, por otro, estaban los trabajadores, en forma de proletarios, libres y urbanizados, que sólo poseían su fuerza de trabajo. Esta condición consolidó una relación de dependencia necesaria para su supervivencia con la nueva clase hegemónica.

Desposeído de todo - excepto de su fuerza de trabajo - el trabajador no tiene otra alternativa que trabajar en las condiciones que se le imponen. Así, mientras la venta sea la regla, como lo es en el capitalismo, ya sea en la producción inmaterial o material, el valor y el trabajo abstracto - creador de valor - no desaparecen como modo de dominación social.

\section{II}

En sus escritos, especialmente en los Fragmentos de las Máquinas, Marx nos da pistas de lo que esperaba de la evolución tecnológica, reflexionando sobre los cambios que la tecnología imprimiría a la naturaleza del capital. De hecho, como hemos afirmado, el mundo del trabajo está sufriendo cambios drásticos, enfrentándose a una nueva era de conflicto entre el capital y el trabajo. Al contrario de lo que podían ofrecer los avances en tecnología y comunicación, Marx ya intuía que la innovación tecnológica, en el capitalismo, no mejoraría la vida concreta de los trabajadores; esto sólo sería posible en otro modo de producción. En los marcos del capitalismo, la innovación tecnológica vino acompañada de un aumento de la productividad, del desempleo y de la consiguiente contención de los salarios frente al creciente ejército de reserva, sin comprometer el beneficio. Dice Marx:

La naturaleza no construye máquinas, ni locomotoras, ferrocarriles, telégrafos eléctricos. Son éstos, productos de la industria humana: material natural, transformado en órganos de la voluntad humana sobre la naturaleza o de su actuación en la naturaleza. Son órganos 
del cerebro humano creados por la mano humana; fuerza objetivada del conocimiento. El desarrollo del capital fixe revela hasta qué punto el conocimiento o conocimiento social general se ha convertido en fuerza productiva inmediata, $y$, por lo tanto, hasta qué punto las condiciones del proceso de la vida social misma han entrado bajo los controles del general intellect y remodeladas conforme al mismo. Hasta qué punto las fuerzas productivas sociales son producidas no sólo en la forma del conocimiento, sino como órganos inmediatos de la práctica social, del proceso vital real

(1972, p. 230)

Cuando pensamos en la digitalización del mundo del trabajo en la actualidad, está claro que es el producto de la evolución de un proceso que abarcó siglos y continentes, y que Marx analizó en profundidad. Es el trabajo humano el que evoluciona frente a estas nuevas tecnologías. Como dijimos, sin plusvalor no puede haber ganancia para valorizar y mantener el dominio del capital. Sin valor-trabajo y plusvalor no puede haber capitalismo. Las formas en que aparecen el trabajo necesario y el excedente cambian, pero el modo de producción y sus bases son las mismas. El capitalismo puede prescindir del empleo; sin embargo, siempre habrá necesidad de vender fuerza de trabajo a otros.

La contradicción capital-trabajo en el mundo digitalizado de hoy, expone nuevos retos a los investigadores, especialmente en lo que algunos autores han llamado la subsunción subjetiva del trabajo al capital (Dardot y Laval, 2013; Mora, 1996) la autogestión subordinada, el auto-emprendimiento o la uberización del trabajo (Abilio, 2018), proporcionada por la centralidad de las plataformas digitales en el control y la gestión del trabajo.

Presenciamos la consolidación de algo que aparece como "nuevo", pero que remite a la plena realización de lo que mueve la contradicción entre el capital y el trabajo desde sus inicios, que es la reducción del trabajador a pura fuerza de trabajo. En este escenario, cada cual debe responsabilizarse de sí mismo, de la gestión de su propia supervivencia. En el mundo de los empresarios de sí mismo, las responsabilidades del Estado y del propio capital para garantizar la reproducción de la fuerza de trabajo se desmoronan y se transfieren al propio trabajador.

Ya no hay garantías sobre el descanso, jornada de trabajo, el envejecimiento, e incluso el acceso a las condiciones mínimas de vida para su reproducción no están asegurados. El trabajador, en este escenario, es movilizado de la manera más eficiente posible para el capital, utilizado cuando es necesario, y luego descartado, para ser utilizado de nuevo en otro momento, consolidando lo que se ha llamado trabajador bajo demanda (Stefano, 2016).

En la autogestión subordinada (Abilio, 2018), típica del trabajo en plataformas digitales, el trabajador pierde todas las garantías sobre lo que recibe y los mínimos para su supervivencia. Además, no hay control sobre la distribución del trabajo y el valor de su tiempo de labor, lo que caracteriza la idea de la uberización del trabajo. Esta situación es una tendencia que atraviesa el mundo laboral de forma global, además de impulsar la eliminación de derechos y trasladar los riesgos y costes de sus actividades al propio trabajador.

\section{III}

Debido a los avances tecnológicos registrados en el último siglo, es posible que personas y objetos, de manera rápida y segura, se trasladen de un continente a otro en pocas horas. Sin embargo, aunque la movilidad aérea sea una posibilidad, el acceso a ella está limitado a quienes pueden pagar por este servicio. Es decir, si bien existe 
la posibilidad de que un individuo se mueva entre continentes y cruce océanos en un corto tiempo, esto está restringido por los imperativos de una economía capitalista, en la que los bienes y servicios producidos socialmente no se dirigen a la satisfacción de las necesidades humanas, sino al enriquecimiento de una minoría de la población, la clase dominante, o sea, la burguesía.

Del mismo modo, en virtud de los avances tecnocientíficos, la vacuna contra el virus Sars-Cov-2 se produjo en tiempo récord durante la pandemia que se inició en 2020. Aun así, al observar los datos relacionados con la vacunación hasta el $1^{\circ}$ de julio de 2021, está claro que, si bien Estados Unidos de América y la Unión Europea han vacunado a más del $50 \%$ de su población con al menos una dosis, el continente africano tiene solo el 2,68\% de su población total vacunada ${ }^{1}$. Esa discrepancia en cuanto a la tasa de vacunación demuestra una de las varias expresiones del carácter desigual del capitalismo: el acceso a los productos de la ciencia y de la tecnología.

De estos simples ejemplos se desprende una lección importante: bajo el capitalismo, el avance tecnocientífico, que es producto del conocimiento de hombres y mujeres, se convierte en mercancía. Además, este proceso de mercantilización no tiene como objetivo satisfacer los intereses colectivos, sino los intereses privados de la clase dominante. Por ello, esta contradicción no es fortuita, sino inmanente al modo de producción capitalista, cuyas bases son la propiedad privada y la obtención de plusvalor por medio de la explotación del trabajo. En síntesis, el avance tecnológico desarrollado en las sociedades capitalistas no se separa de las relaciones sociales burguesas en las que se produce, hecho que explica el acceso desigual a bienes y servicios tecnocientíficos, como, por ejemplo, las vacunas contra el Covid-19 e, incluso, tratamientos médicos y hospitalarios.

No obstante, admitir ese contexto sociopolítico no implica asumir una crítica romántica y reaccionaria, centrada únicamente en los aspectos negativos del desarrollo tecnológico. Del mismo modo, es igualmente equivocado adoptar cualquier tipo de idolatría del progreso. Por ello, en oposición a esas ideas simplificadoras de la realidad, se admite que, bajo el capitalismo, la tecnología adquiere un carácter contradictorio, pudiendo constituirse como un baluarte del progreso o de la barbarie. Es decir, además del problema del acceso desigual, el conocimiento tecnocientífico puede servir tanto para la creación de vacunas como para la creación de tecnologías de control y exterminio, como las que utilizó el Estado nazi alemán durante el siglo XX o como las que se utilizan en ocupaciones neocoloniales (Espinoza, 2018; Wilcox, 2016).

El carácter dialéctico del desarrollo tecnológico revela que, aunque la ciencia y la tecnología tengan el potencial de promover mejoras en la calidad de vida de las poblaciones globales, el avance tecnocientífico, en la sociedad capitalista, además de estar distribuido de manera desigual, se incorpora a fin de cumplir los intereses del capital, construyendo nuevas tecnologías de control (Lemos, Galindo, Natale, Silva, y Santos, 2014; Wilcox, 2016), desarrollando nuevas formas de explotación laboral por plataforma (Antunes, 2021; Papadimitropoulos, 2021; Slee, 2019) y expandiendo la producción de plusvalor en su forma relativa a través del aumento de la productividad (Marx, 1973).

\section{IV}

Por tanto, uno de los desafíos que se plantean a nivel teórico-práctico, es decir, de la praxis, es retirar la ciencia y la tecnología del dominio del capital, de modo que no más se condicionen a los imperativos del lucro, sino a las necesidades de los seres humanos. Hacerlo, sin embargo, implica la inevitable confrontación con el capitalismo. En este artículo nos gustaría ensayar algunas reflexiones sobre una de las formas de

\footnotetext{
${ }^{1} 1$ Según las estadísticas disponibles en el sitio web Our World in Data.
} 
construcción de este enfrentamiento: la que ocurre mediante el proceso formal de enseñanza-aprendizaje, con especial énfasis en las instituciones de educación superior.

De ninguna manera se pretende acercarse a una concepción idealista del proceso de enseñanza-aprendizaje, tratándolo como una panacea. Sin embargo, al intentar escapar de las trampas del idealismo, hay que tener cuidado de no dejarse confundir por una noción fatalista, que rechaza la educación como una mediación importante, ya sea en el proceso de transformación social o en el mantenimiento del status quo. Por ello, el enfoque aquí presentado se centrará en una manera específica a través de la cual es posible reformar el currículo universitario a fin de considerar el debate sobre la ética y el interés social de la producción científica y tecnológica.

Evidentemente, es necesario reconocer que la disputa por la producción de una ciencia comprometida con el bienestar social no termina con la reformulación de los currículos universitarios, puesto que articular el desarrollo tecnocientífico con los intereses de la clase obrera presupone poder político y capacidad de decisión en la definición de las políticas públicas y del sector económico. Pero, aun así, esta circunstancia no anula la importancia del papel de las universidades, instituciones que, además de capacitar los profesionales de cualquier sociedad, poseen la función de garantizar las condiciones necesarias para el desarrollo de la ciencia, la tecnología, la filosofía y la teoría social.

Como señalan Junior, Deconto, Neto, Cavalcanti, y Ostermann (2014) y Linsingen (2007), el debate sobre la relación entre ciencia, tecnología y sociedad en los planes de estudios universitarios se remonta a las décadas de 1960 y 1970. Y, aunque diverso, este movimiento convergió en torno a la crítica a la noción de que ciencia y tecnología están desvinculados de las relaciones sociales capitalistas. Por ello, el citado período permitió un ambiente en que se desarrollaran fructíferas discusiones acerca de la construcción de una ética, es decir, una forma de actuar que pudiera servir de guía para que los científicos y los ingenieros produjeran una práctica profesional con interés social, $\mathrm{o}$, al menos, se posicionaran de manera reflexiva ante sus trabajos.

Además, en conformidad con la perspectiva defendida por Junior et al. (2014), se asume que la adopción de una mirada marxista en los debates sobre ciencia, tecnología y sociedad constituye un marco teórico importante para enfrentar concepciones que abogan la ciencia y la tecnología como neutrales y autónomas. Aunque el tema sea desarrollado en la obra marxiana de manera dispersa y secundaria, los fundamentos de su teoría social, como el pensamiento dialéctico, la perspectiva de la lucha de clases y la subsunción del trabajo al capital proporcionan las herramientas teóricas necesarias para reflexionar de forma crítica e inventiva sobre el modo como aparece y se utiliza la tecnología en la sociedad burguesa.

Así mismo, la reflexión en las universidades sobre ciencia, tecnología y sociedad debe propiciar debates acerca del papel de las tecnologías en la reforma de la dinámica laboral. Pues, entre las diversas metamorfosis registradas en la etapa actual del capitalismo, se destaca un desplazamiento desde el entorno laboral al domicilio por medio de los smartphones y aplicaciones de mensajería instantánea, lo que contribuye directamente para la superexplotación del trabajo y la reducción de la calidad de vida de la clase obrera.

En este sentido, corresponde a la universidad, como entorno institucional para el desarrollo del pensamiento, posicionarse sobre estos temas, prontamente encubiertos por la ideología liberal con discursos como el que afirma que las nuevas tendencias de precariedad de la vida son el "nuevo normal" y, por eso, corresponde al sujeto explotado adaptarse. El rápido desarrollo de las tecnologías de comunicación, especialmente a través de Internet, así como las metamorfosis en el mundo del trabajo, no permitieron el tiempo suficiente para una educación adecuada sobre los usos de estas tecnologías y cómo ellas pueden ser captadas por las empresas y por el mercado, contribuyendo para el agotamiento físico y psíquico de los trabajadores y trabajadoras. 
Si, por un lado, los últimos años registraron el aumento paulatino de una amplia bibliografía acerca de los efectos nocivos del empleo tecnológico en el mundo del trabajo bajo la égida del capital (Antunes, 2018, 2020; Antunes y Braga, 2009), los efectos del uso exacerbado de tecnologías de comunicación y redes sociales, por ejemplo, aún no se encuentran plenamente documentados, aunque existan registros sobre los efectos provocados en el ámbito físico y psicológico, e incluso la dependencia, tanto en adultos como en niños.

Además del objetivo de conectar a las personas, las redes sociales también han adquirido funciones no declaradas, que poseen centralidad en la experiencia digital contemporánea. Aunque no sea explícitamente asumido por los usuarios, el patrón de comportamiento en las redes sociales indica que ellas son, en gran parte, herramientas de autoafirmación y proclamación de valores ampliamente difundidos como virtuosos por la racionalidad capitalista: éxito material, expresión del culto de la propiedad privada; y, el refuerzo de una concepción estética ideal, que rechaza la diversidad humana. Evidentemente, el universo de las redes sociales no se constituye como un bloque monolítico, pero la experiencia contemporánea, marcada por la profusión de influencers y coaches digitales, apunta a una tendencia hacia la "espectacularización del yo", convergiendo en la construcción de un modelo ideal de sujeto neoliberal, para emplear el concepto de Dardot y Laval (2013).

En conjunto con esas características, el desarrollo de las tecnologías de información y la obtención de datos de usuarios han permitido la creación de algoritmos que identifican las preferencias de quienes utilizan las redes sociales, o que, a su vez, posibilitó configurar la experiencia digital de manera a provocar la necesidad de dedicarse cada vez más tiempo frente a la pantalla de los dispositivos electrónicos. Además, en un contexto marcado por la privación de oportunidades y las incertidumbres acerca del futuro, el uso de las redes sociales, así como el acto de "revisar el feed" o "escrolear", de manera casi automática, brindan una dosis de placer para el trabajador contemporáneo, quien, en tiempos de avance del capitalismo neoliberal, tiene cada vez más limitadas las posibilidades de ejercitar sus potencialidades y, con ello, realizarse. Por tanto, el uso de las redes sociales y, no menos importante, el uso excesivo de los smartphones, también figuran como mecanismos de escape de una sociabilidad que amarga los efectos cada vez más intensos de la precarización neoliberal de las condiciones generales de vida.

De este modo, si los efectos del uso de las tecnologías digitales son perjudiciales a los adultos, ellos son aún más acentuados para los niños, debido a la particular condición de desarrollo de estos sujetos. Por tanto, existe la necesidad de una formación académica que esté atenta a esos temas, con el fin de formar profesionales capaces de desarrollar, de forma transversal, una educación que, sin moralismos ni idealismos, permita el florecimiento de un proceso de reflexión sobre las posibilidades de construir una relación sana con las diversas expresiones de la tecnología.

Los smartphones, bienes que se han convertido en objeto de deseo entre niños, adolescentes y jóvenes, les aparecen como naturales, prácticamente como apéndices del cuerpo humano, máquinas necesarias para la supervivencia moderna. En este sentido, al tratar su existencia y usos como banales, la reflexión sobre las maneras como se utilizan esos dispositivos acaba siendo relegada y, con ello, se contribuye para que la relación sujeto-máquina se invierta en una relación fantasmagórica, en la que la máquina domina al sujeto.

\section{CONSIDERACIONES FINALES}

Por tanto, conforme desarrollado al largo de este artículo, la digitalización en el mundo del trabajo y el avance de la tecnología vienen acompañados de la precariedad de las condiciones de vida de la clase obrera. Por un lado, los trabajadores se están reduciendo 
a trabajadores informales y, por otro, los mecanismos de gestión y control de los procesos de trabajo están perdiendo sus formas socialmente reconocibles y estables. En otras palabras, en este modelo, se trabaja más y no hay prescripciones formales sobre el trabajo.

Es necesario un compromiso permanente de uno mismo. Esta situación, aunque con nuevas formas, no difiere de las condiciones que el capitalismo periférico impone a innumerables trabajadores que necesitan, en un movimiento constante y permanente, tejer su reproducción individual y social en condiciones estructurales que nunca les han dado garantías. Para Marx, la tecnología abrió nuevos paradigmas, pero la emancipación del trabajo asalariado tenía que estar relacionada con el control de la técnica, que no es neutral, y siempre ha estado al servicio del capital.

Más allá de las implicaciones de los usos de la tecnología a fin de cambiar las relaciones capital-trabajo en beneficio de la clase dominante, el uso de las redes sociales y el vínculo entre humanos, máquinas y tecnología de datos posee papel fundamental en el mantenimiento y en la consolidación de estructuras psíquicas asociadas al sostenimiento del capitalismo.

Por ello, dada la centralidad que juegan las tecnologías digitales en la experiencia humana contemporánea, es imperativo colocarlas en el centro del debate político, académico y público, con el objetivo de, al mismo tiempo, impulsar el desarrollo tecnocientífico para satisfacer las necesidades humanas y formar ciudadanos informados sobre las formas como pueden utilizar y también ser utilizados por las tecnologías digitales.

\section{REFERENCIAS}

Abilio, L. (2018). Uberización: nuevas formas de control, organización y gestión del trabajo. En O. Cortés y A. Constanza (Eds.), Trabajo y subjetividad. Bogotá, Colombia: Universidad Libre.

Antunes, R. (2018). Parte I - Entre a corrosão e os escombros: o advento do proletariado da era digital. En R. Antunes (Ed.), O privilégio da servidão: o novo proletariado de serviços na era digital. São Paulo: Boitempo.

Antunes, R. (2020). Uberização, trabalho digital e indústria 4.0. São Paulo: Boitempo.

Antunes, R. (2021). O capitalismo de plataforma e a desantropomorfização do trabalho. En R. Grohmann y R. (Eds.), Os laboratórios do trabalho digital: entrevistas (pp. 33-38). São Paulo: Boitempo.

Antunes, R., y Braga, R. (2009). Infoproletários: degradação real do trabalho virtual. São Paulo: Boitempo.

Chesnais, F. (2016). Finance capital today: corporations and banks in the lasting global slump. Boston: Brill Academic.

Dardot, P., y Laval, C. (2013). La nueva razón del mundo. Ensayo sobre la sociedad neoliberal. Barcelona: Gedisa.

Espinoza, M. (2018). State terrorism: orientalism and the drone programme. Critical Studies on Terrorism, 11(2), 376-393. https://doi.org/10.1080/17539153.2018.1456725

Junior, P. L., Deconto, D. C. S., Neto, R. A., Cavalcanti, C. J. H., y Ostermann, F. (2014). Marx como referencial para análise de relações entre Ciência, Tecnologia e Sociedade. Ciência \& Educação, 20(1), 175-194.

Lemos, F. C. S., Galindo, D., Natale, A., Silva, D. G., y Santos, I. C. (2014). A guerra atual e o uso de drones: práticas biopolíticas do matar em nome da vida. Revista Psicologia Política, 14(30), 283-295.

Linsingen, I. V. (2007). Perspectiva educacional CTS: aspectos de um campo em consolidação na América Latina. Ciência \& Ensino, 1, 1-19. Descargado de https://wiki.sj.ifsc.edu.br/ images/2/23/Irlan.pdf 
Lukács, G. (1985). El joven Hegel y los problemas de la sociedad capitalista. Barcelona: Grijalbo.

Lukács, G. (2004). Ontología del ser social: el trabajo. Buenos Aires: Herramienta.

Marx, K. (1972). Elementos fundamentales para la crítica de la economía política. México: Siglo XXI.

Marx, K. (1973). El Capital. Crítica de la economía política (Vol. I). México: FCE.

Marx, K., y Engels, F. (2014). La ideología alemana. Madrid: Akal.

Mészáros, I. (2009). A Crise Estrutural do Capital. São Paulo: Boitempo Editorial.

Mora, H. (1996). Modernización capitalista y trabajo abstracto: ¿sociedad pos capitalista o subsunción real del trabajo general? En. Revista Economía y Sociedad, 1(2).

Descargado de https://www.revistas.una.ac.cr/index.php/economia/article/view/6852

Papadimitropoulos, E. (2021). Platform Capitalism, Platform Cooperativism, and the Commons. Rethinking Marxism, 33(2), 246-262.

Schmidt, A. (1977). El concepto de naturaleza en Marx. Madrid: Siglo XXI.

Slee, T. (2019). Uberização: a nova onda do trabalho precarizado. São Paulo: Editora Elefante.

Stefano, V. D. (2016). The rise of the "just-in-time workforce": On-demand work, crowdwork and L. Kostek Abilio [ 11 ] labour protection in the "gig-economy". Comparative Labor Law \& Policy fournal, 71.

Wilcox, L. (2016). Embodying algorithmic war: Gender, race, and the posthuman in drone warfare. Security Dialogue, 48(1), 11-28. https://doi.org/10.1177/0967010616657947

Wittmann, D. (2009). Las fuentes del concepto de alienación. En E. Renault (Ed.), Los Manuscritos de 1844 de Marx (pp. 103-124). Buenos Aires: Nueva Visión. 